Nota científica

(Short communication)

\title{
REGISTROS RECIENTES DE FELINOS EN EL BOSQUE DE NIEBLA EN SANTIAGO COMALTEPEC, SIERRA NORTE DE OAXACA, MÉXICO
}

\author{
RECENT RECORD OF BIG CATS IN CLOUD FOREST OF SANTIAGO \\ COMALTEPEC, SIERRA NORTE OF OAXACA, MEXICO
}

\section{Mayra Karelly ESPINOZA-RAMÍREZ, ${ }^{1}$ María Delfina LUNA-KRAUleTZ, ${ }^{2, *}$ Cecilia ALFONSO-CORRADO ${ }^{2}$ Y RICARDO CLARK-TAPIA ${ }^{2}$}

\author{
${ }^{1}$ Maestría en Ciencias en Innovación en Manejo de Recursos Naturales. Colegio de Postgraduados Campus San \\ Luis Potosí, México <aryam_yllerak@hotmail.com>. \\ ${ }^{2}$ Universidad de la Sierra Juárez. Avenida Universidad s/n. Ixtlán de Juárez, Oaxaca. C.P. 68725, México \\ <mkrauletz@unsij.edu.mx>, <liana@unsij.edu.mx>y<rclark@unsij.edu.mx>. \\ * Autor de correspondencia: <mkrauletz@unsij.edu.mx>. \\ Recibido: 28/04/2016; aceptado: 23/ 02/2017 \\ Editor responsable: Vinicio Sosa Fernández.
}

\begin{abstract}
Espinoza-Ramírez, M. K., Luna-Krauletz, M. D., Alfonso Corrado, C. y Clark-Tapia, R. (2017). Registros recientes de felinos en el bosque de niebla en Santiago Comaltepec, Sierra Norte de Oaxaca, México. Acta Zoológica Mexicana (n.s.), 33(2), 398-401.
\end{abstract}

RESUMEN. Se confirma la presencia de cuatro grandes felinos, Panthera onca, Puma concolor, Leopardus pardalis y Leopardus wiedii, en el bosque de niebla de la comunidad La Esperanza, Santiago Comaltepec, Oaxaca. Se usó fototrampeo para evaluar la presencia de estas especies en dos transectos de 2.5 y $1.5 \mathrm{~km}$ respectivamente, desde agosto de 2014 a febrero de 2016. Esta información es necesaria para la conservación in situ de estas especies.

La familia Felidae está representada en México por seis especies: jaguar (Panthera onca, Linnaeus 1758), puma (Puma concolor, Linnaeus 1771), jaguarundi (Puma jaguaroundi, E. Geofroy 1803), ocelote (Leopardus pardalis, Linnaeus 1758), tigrillo (Leopardus wiedii, Schinz 1821) y gato montés (Lynx rufus, Schreber, 1777) (Ceballos \& Oliva, 2005). La NOM-059-SEMARNAT-2010 categoriza en peligro de extinción a $P$. onca, $L$. pardalis y L. wiedii. En México, las especies de la familia Felidae se distribuyen en diversos ecosistemas como son bosques de coníferas, bosques tropicales y bosques de niebla (Charre-Medellín 2014; Buenrostro-Silva et al., 2015; CarreraTreviño et al., 2016). Para el Estado de Oaxaca, destacan los registros de P. onca (Figel et al., 2009; Briones et al.,
Espinoza-Ramírez, M. K., Luna-Krauletz, M. D., Alfonso Corrado, C., \& Clark-Tapia, R. (2017). Recent record of big cats in cloud forest of Santiago Comaltepec, Sierra Norte of Oaxaca, Mexico. Acta Zoológica Mexicana (n.s.), 33(2), 398-401.

ABSTRACT. We confirm the presence of four big cats, Panthera onca, Puma concolor, Leopardus pardalis and Leopardus wiedii in cloud forest on La Esperanza community lands, Santiago Comaltepec, Oaxaca. Camera traps were used to evaluate the presence of these species in two transects of $2.5 \mathrm{~km}$ and $1.5 \mathrm{~km}$ respectively, from August of 2014 to February of 2016. This information is necessary for the conservation in situ of these species.

2012), P. concolor (Lavariega et al., 2012), L. pardalis (Meraz et al., 2010), y L. wiedii (Cinta-Magallón et al., 2012). Estas especies son importantes en la estructura trófica del ecosistema (Steneck, 2005), por lo que su presencia como especies reguladoras de la densidad poblacional de otras especies, es imprescindible para que no se afecte la dinámica, estructura y función del bosque.

A pesar de la importancia ecológica y de los registros que se tienen sobre las especies de felinos en Oaxaca, aún existen zonas no exploradas, y por lo tanto se carece del conocimiento biológico necesario para proponer medidas de conservación in situ a través de herramientas claves (e.i reservas comunitarias, corredores biológicos, entre otros ). En este contexto, es imprescindible continuar 
realizando inventarios locales de especies de felinos, ya que es básico para la toma de decisiones de proyectos de manejo y conservación de recursos naturales (Lavariega et al., 2012); particularmente en la región Sierra NorteChinantla donde se encuentran los bosques de niebla más extensos y conservados de México (Anta-Fonseca et al., 2010). En el presente trabajo se reportan registros recientes de cuatro especies de felinos mediante la técnica de fototrampeo en el bosque de niebla de la localidad La Esperanza, Santiago Comaltepec, Sierra Norte, Oaxaca.

El muestreo se realizó durante los meses de agosto del 2014 a febrero del 2016 en dos sitios del bosque de niebla de la Comunidad de Santiago Comaltepec, conocidos localmente como El Orquideario (14Q 777611 E y 194893 N) y Ruta San Bernardo (14Q 779655 E y 1943950 N), ambos sitios con Datum de referencia WGS84 y resolución de 15 m (Fig. 1). La altura promedio de las zonas es de $1786 \mathrm{msnm}$ y la precipitación promedio anual se reporta entre 5000 a 6000 mm (Gual-Díaz \& GonzálezMedrano, 2014).

Se ubicaron un total de 10 estaciones simples de monitoreo, cinco sobre el sendero Ruta San Bernardo y cinco en el sendero El Orquideario. Se utilizaron 10 fototrampas marca Cuddeback ${ }^{\circledR}$ Attack con resolución de 5.0 megapixeles, y fueron ubicadas de manera permanente sobre los senderos a una altura aproximada de 50 a 80 $\mathrm{cm}$ del suelo dependiendo de las condiciones topográficas de cada sitio, la separación entre cámaras osciló entre 0.5 y $1 \mathrm{~km}$ y todas permanecieron activas durante las 24 h, sumando un esfuerzo total de muestreo de 2,920 días
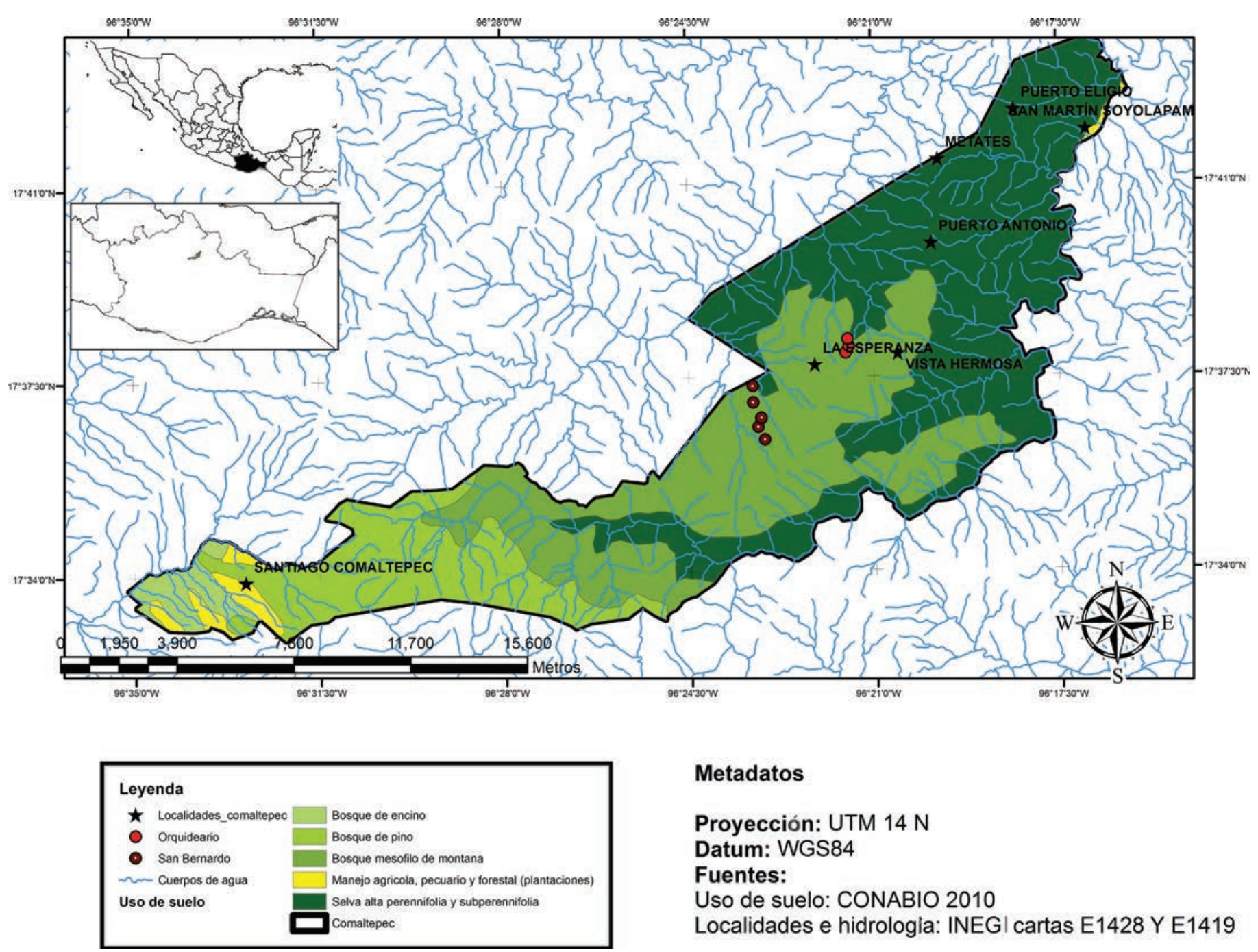

\section{Metadatos}

\section{Proyección: UTM $14 \mathrm{~N}$ \\ Datum: WGS84}

Fuentes:

Uso de suelo: CONABIO 2010

Localidades e hidrologia: INEGI cartas E1428 Y E1419

Figura 1. Área de Estudio en La Esperanza, Santiago Comaltepec, Sierra Norte de Oaxaca, México. 
trampa. Los resultados obtenidos fueron 32 fotocapturas independientes, de las cuales 19 corresponden a $P$. concolor, ocho a $P$. onca, cuatro a $L$. wiedii y un registro para L. pardalis (Fig. 2). De los 19 registros de $P$. concolor se pueden inferir al menos dos individuos diferentes por el tamaño de los ejemplares fotografiados; de acuerdo al patrón de manchas se registraron dos individuos de jaguar, un tigrillo y un ocelote. En cuanto al patrón de actividad registrado para $P$. concolor, los registros variaron ampliamente durante las 24 horas, y no presentaron un patrón de horario claramente definido. $P$. onca presentó un horario de actividad tanto diurno como nocturno, predominando un mayor número de registros a partir de las 16:00 h has- ta las 19:00 h. El tigrillo fue fotografiado a las 04:16 h, 06:21 h, 13:33 h y las 21:51 h. El ocelote únicamente se fotografió a las 03:42 h.

Estos registros representan una aportación importante al conocimiento de la diversidad en el bosque de niebla y se continuará con el monitoreo. La presencia y coexistencia de cuatro depredadores en una misma zona puede deberse a una alta disponibilidad de presas. Los registros fotográficos mostraron la presencia de seis presas potenciales, y cuyo número de evidencias fue variable: Sciurus aureogaster (46), Didhelpis virginiana (40), Crax rubra (10), Pecari tajacu (9), Mazama temama (1) y Dasypus novemcintus (1).
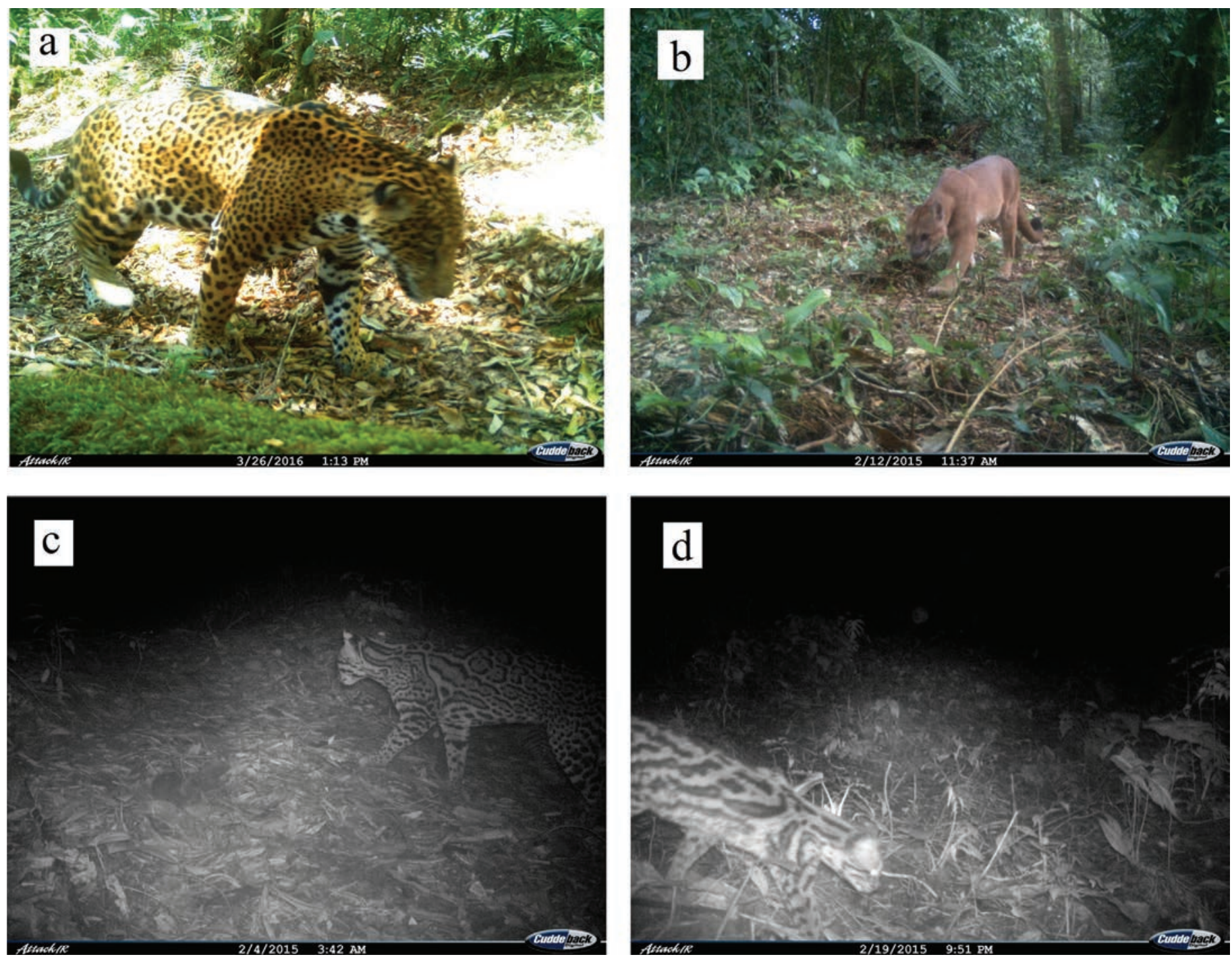

Figura 2. Felinos registrados en el bosque de niebla en La Esperanza, Santiago Comaltepec, Sierra Norte de Oaxaca. a) Panthera onca, b) Puma concolor, c) Leopardus pardalis y d) Leopardus wiedii. 
Los datos obtenidos en este trabajo permitirán fortalecer las estrategias de conservación bajo enfoque comunitario en Santiago Comaltepec, que desde el año 2004 cuenta con un decreto interno de refugio para la vida silvestre incorporado en su Plan de Ordenamiento Territorial comunitario.

AGRADECIMIENTOS. Al Consejo de Ciencia y Tecnología del Estado de Oaxaca, mediante el proyecto: Fondo Mixto 195425-UNSIJ. Al Consejo Nacional de Ciencia y Tecnología (CONACYT CVU 635918) por la beca de postgrado otorgada al primer autor. A la Autoridad Agraria de Santiago Comaltepec por su apoyo en la realización de este estudio. Finalmente las observaciones y valiosos comentarios de dos revisores anónimos ayudaron a mejorar en gran medida el presente trabajo.

\section{LITERATURA CITADA}

Anta-Fonseca S., Galindo-Leal, C., González Medrano, F., Koleff-Osorio, P., Meave del Castillo, J., Moya-Moreno, H. \& Victoria-Hernández, A. (2010). IX. Sierra Norte de Oaxaca. En: Toledo-Aceves, T. (Coord.). Comisión Nacional para el Conocimiento y Uso de la Biodiversidad (CONABIO) Eds. El Bosque Mesófilo de Montaña en México: Amenazas y oportunidades para su conservación y su manejo sostenible. CONABIO, México, 1081115 pp.

Briones-Salas, M., Lavariega, M. C. \& Lira, I. (2012). Distribución actual y potencial del jaguar (Panthera onca), en Oaxaca, México. Revista Mexicana de Biodiversidad, 83, 246-257.

Briones-Salas, M., Sánchez-Cordero, V. \& Quintero, C. (2001). Listado de mamíferos terrestres del norte de Oaxaca. Anales del Instituto de Biología, UNAM, Serie Zoológica, 72, 125-161.

Buenrostro-Silva, A., Sigüenza-Pérez, D. \& García Grajales, J. (2015). Mamíferos carnívoros del parque Nacional Lagunas de
Chacahua, Oaxaca, México: Riqueza, abundancia y patrones de actividad. Revista Mexicana de Mastozoología nueva época, 5, 39-54.

Carrera-Treviño, R., Cavazos, J. J., Briones-Salas, M. \& Lira-Torres, I. (2016). Registro actual del jaguar Panthera onca (Carnivora: Felidae) en el Parque Nacional Cumbres de Monterrey, Nuevo León, México. Revista Mexicana de Biodiversidad, 87, 270-275.

Ceballos, G. \& Oliva, G. (2005). Los mamíferos silvestres de México. Fondo de Cultura Económica. CONABIO, México, 986 pp.

Charre-Medellín, J. F., Monterrubio-Rico, T., Guido-Lemus C. D. \& Mendoza, E. (2015). Patrones de distribución de felinos silvestres (Carnivora: Felidae) en el trópico seco del Centro-Occidente de México. Revista de Biología Tropical, 63, 783-797.

Cinta-Magallón, C. C., Bonilla-Ruz, C. R., Alarcón-D., I. \& Arroyo-Cabrales, J. (2012). Dos nuevos registros de margay (Leopardus wiedii), en Oaxaca, México con datos sobre hábitos alimentarios. Cuadernos de Investigación UNED, 4, 33-40.

Figel, J. J., Durán, E., Bray, D. B., \& Prisciliano, J. R. (2009). New jaguar records from montane forest at a priority site in southern Mexico. CATnews, 50,14-15.

Gual-Díaz, M. \& Gónzalez-Medrano, F. (2014). Los bosques mésofilos de montaña en México. Pp. 27-67. In: M. Guál-Diáz \& Rendón-Correa, A. (Eds.) Bosques Mésofilos de Montaña de México: diversidad, ecología y manejo. CONABIO, México.

Lavariega, M. C., Briones-Salas, M. \& Gómez-Ugalde, R. M. (2012). Mamíferos medianos y grandes de la Sierra de Villa Alta, Oaxaca, México. Maztozoología Neotropical, 19, 225-241.

Morales-García, J. J., Morales-García, A. D. \& Acosta-Rosales, A. (2015). Registros recientes de jaguar (Panthera onca) en el estado de Hidalgo, México. Revista Mexicana de Mastozoología nueva época, 5, 66-72.

Steneck, R. S. (2005). An ecological context for the role of large carnivores in conserving biodiversity. Pp. 9-33 In: Ray, J. C., K. H. Redford, R. S. Steneck, \& J. Berger (eds.). Large carnivores and the conservation of biodiversity. Island Press. Washington, EE.UU. 